\title{
Optimal Selection of Customers for a Last-Minute Offer
}

\author{
Roberto Cominetti \\ Departamento de Ingeniería Matemática, Universidad de Chile, Santiago, Chile, rcominet@dim.uchile.cl \\ José R. Correa \\ Departamento de Ingeniería Industrial, Universidad de Chile, Santiago, Chile, jcorrea@dii.uchile.cl \\ Thomas Rothvoß \\ Institute of Mathematics EPFL, Lausanne, Switzerland, thomas.rothvoss@epfl.ch \\ Jaime San Martín \\ Departamento de Ingeniería Matemática and Centro de Modelamiento Matemático, Universidad de Chile, \\ Santiago, Chile, jsanmart@dim.uchile.cl
}

\begin{abstract}
We analyze a short-term revenue optimization problem involving the targeting of customers for a promotion in which a finite number of perishable items are sold on a last-minute offer. The goal is to select the subset of customers to whom the offer will be made available in order to maximize the expected return. Each client replies with a certain probability and reports a specific value that might depend on the customer type, so that the selected subset has to balance the risk of not selling all items with the risk of assigning an item to a low value customer.

We show that threshold strategies, which select all those clients with values above a certain optimal threshold, might fail to achieve the maximal revenue. However, using a linear programming relaxation, we prove that they attain a constant factor of the optimal value. Specifically, the achieved factor is $1 / 2$ when a single item is to be sold and approaches 1 as the number of available items grows to infinity. Also, for the single item case, we propose an upper bound based on a sharper linear relaxation that allows us to obtain a threshold strategy achieving at least $2 / 3$ of the optimal revenue. Moreover, although the complexity status of the problem is open, we provide a polynomial time approximation scheme for the single item case.
\end{abstract}

Subject classifications: promotional sale; linear programming relaxations; approximation algorithms.

Area of review: Optimization.

History: Received May 2007; revisions received December 2008, May 2009; accepted September 2009. Published online

in Articles in Advance February 26, 2010.

\section{Introduction}

In this paper we consider a short-term revenue optimization problem that involves selecting a set of customers for a promotion in which a finite number of perishable items are sold on a last-minute offer. The goal is to maximize the total expected return by choosing the subset of customers to whom the offer will be made available, taking into account that each client replies with a certain probability and reports a specific value that might depend on the customer type. Any item not sold is either lost or derived to a secondary market at a fixed price that sets a reference value from which we measure a customer's surplus value.

As a motivation example consider the situation faced by an airline offering discount business upgrades on a flight close to its departure date, say two days ahead. The offer may be sent to some or all of the passengers that hold a current booking in economy class for that flight. The airline values its clients differently, based on personal characteristics such as past travel behavior, propensity to buy an upgrade at regular price, level of fidelization, or other specific demographic data. However, because of lack of time it is not possible to make the offer on a one-by-one basis from higher to lower valued customers, and they must all be contacted at once by e-mail, after which the items will be assigned according to a first-come-first-served discipline.

A similar situation happens with the business class upgrades sold at the gate by several airlines. Available business class seats are offered at a certain price to a subset of the customers who are waiting for boarding, who may be discriminated according to the fare class they bought. Although the price is flat, the airline values its clients differently, as consistently making upgrades available for cheaper fares disincentives the sales for more expensive ones. If, however, the upgrades are made available only to the highest fare class, the airline risks of not selling enough upgrades. One can imagine similar situations in the car or computer industries, where a company wants to put on sale some old units currently held in stock before a new model is released to the market. 
Intuitively, the promotion should include enough customers to have a high probability of selling all the items. However, if too many customers are included, one may face an excess of demand and end up selling an item to a low revenue client who displaces a higher valued customer responding later. Handling this type of trade-off is a basic issue in revenue management problems. A natural policy is to select all customers whose values are above a certain threshold, which is chosen to maximize the expected revenue. Such strategies are indeed optimal if low revenue clients react to the promotion before higher revenue clients but could fail to be optimal otherwise. In our short-term setting, we assume that there is no correlation between the order in which customers react and their value. In this case we show that a threshold policy, although not optimal, attains a guaranteed fraction of the optimal revenue. Moreover, this fraction becomes asymptotically close to one as the number of items grows.

In practice, the estimation of the acceptance probabilities and the values of clients might be based on historic data accumulated from previous promotional sales, possibly by grouping the clients into classes or categories according to different demographic and behavioral characteristics. Because such estimates might be imprecise, it is worth noting that, although small errors could significantly change the optimal solution, the optimal objective value varies continuously with the input data. Furthermore, threshold policies are robust under calibration errors because they depend only on the order of clients rather than on specific values. Then our approximation results for these types of policies hold even under noisy data.

Our setting differs significantly from classic revenue management approaches (we refer the reader to Talluri and van Ryzin 2005 for a detailed treatment). Probably the most relevant difference comes form our short notice assumption. For instance, without this assumption the so-called nested policies (i.e., threshold policies applied over several rounds) turn out to be optimal (Ball and Queyranne 2009). On the other hand, although sampling targets from a population for a direct mail campaign is an issue already considered in marketing (Bult and Wansbeek 1995), our model deviates from the marketing literature because the shortnotice assumption does not allow a dynamic approach. In this sense, note that the first-come-first-served discipline appears to be reasonable in a short-notice setting, as it is indeed the right model whenever the response times of the customers are independent and identically distributed. This contrasts with rationing policies that yield better revenues when more time is available to sell the items.

The paper is structured as follows. Section 2 gives a mathematical description of the problem, which leads to a basic, though unexplored, discrete optimization problem. We present in $\$ 2.2$ counterexamples to some natural properties that one might expect, such as the nonoptimality of threshold policies, while in $\S 3$ we show that these types of solutions lead to algorithms with explicit bounds on the approximation guarantees. In particular, we describe a trivial heuristic with a 1/2-approximation guarantee for the single item case that becomes asymptotically optimal when the number of items on sale goes to infinity. In $\S 4$ we discuss a sharper LP relaxation and derive an improved 2/3approximation algorithm for the single item case, which is also a threshold strategy. Then, in $\S 5$, we deviate from threshold strategies and provide an algorithm that takes any $\varepsilon>0$ and outputs a $(1+\varepsilon)$-approximate solution in time, which is polynomial for fixed $\varepsilon$, that is to say a polynomial time approximation scheme (PTAS). We conclude in $\S 6$, pointing out some directions for further research, while in the appendix we present statistics on the numerical performance of the proposed algorithms and their comparison with two additional heuristics.

\section{Problem Formulation}

A finite number of $m$ identical perishable items is to be offered on a promotional sale among a finite set of customers $C=\{1, \ldots, n\}$. Each customer $i \in C$ is characterized by a probability $p_{i} \in[0,1]$ of accepting the offer, and a value $v_{i} \geqslant 0$ that he reports when buying an item. This value represents the net expected revenue, namely, the promotional price minus the expected value of the customer if he is excluded from the promotion, accounting for the possibility that he would buy an item anyway at the regular price. We assume that any unsold item is lost, as for instance in the case of empty seats on a given flight close to its departure date. We also suppose $\sum p_{i} \geqslant m$ and that customers have been pre-ordered by decreasing values so that $v_{1} \geqslant v_{2} \geqslant \cdots \geqslant v_{n}$. The problem is to choose the set of customers $A \subseteq C$ to whom the promotional sale will be made available. If $m$ is large, more clients should be included; while for small $m$, one should be more selective to avoid high-revenue customers being displaced by lowrevenue clients. The goal is to balance the risk of displacement and the risk of not selling all the items, maximizing the total expected return.

Note that we assume neither that the values $v_{i}$ are similar in any way, nor that they are correlated with the probabilities $p_{i}$. Thus the model is also suitable to incorporate other customer-specific characteristics in other type of promotional sales, including regional considerations, loyalty issues, dispatching costs, among others. In some situations, it might be reasonable to assume that $p_{i}>p_{j}$ whenever $v_{i}>v_{j}$. If this is the case, it is easy to observe that threshold policies (i.e., policies selecting all customers reporting a value above a certain threshold) are optimal. However, this assumption is not necessarily the most natural, because $v_{i}$ is the value of the client from the point of view of the company, not the valuation from the point of view of the customer. For instance, consider again the business upgrade example given in the introduction, and assume the value of a client for the company is given by the discounted price of the upgrade minus the regular price times the probability a 
customer buys at regular price. In such a situation, a customer with low propensity to buy (small $p_{i}$ ) will also be less likely to induce dilution (i.e., his propensity to buy at regular price if he does not receive the offer will also be small), and therefore the value of such a client from the point of view of the company will be higher.

\subsection{The Optimization Problem}

To state the problem precisely we introduce decision variables $x_{i} \in[0,1]$, which represent the probability with which we make the offer available to customer $i$. This approach involves a randomized choice of the customers and covers the possibility of an include/exclude policy represented by the extreme values $x_{i}=1$ or $x_{i}=0$. Alternatively, we consider as decision variable the value $y_{i}=p_{i} x_{i}$, which represents the probability for customer $i$ to be a respondent, that is to say, a client who receives the offer and is willing to accept it. Consider the Bernoulli random variables

$Y_{i}= \begin{cases}1 & \text { if customer } i \text { is a respondent } \\ 0 & \text { otherwise }\end{cases}$

with $\mathbb{P}\left(Y_{i}=1\right)=y_{i}$, and let $R=\left\{i \in C: Y_{i}=1\right\}$ be the random set of respondents. If $|R| \leqslant m$ all the demand can be satisfied yielding a total revenue of

$v_{R}=\sum_{j \in R} v_{j}$.

Otherwise, because only $m$ items are available, we must choose $m$ customers from $R$ to assign the items. If this is done according to a first-come-first-served discipline and we assume no correlation between the $\left(p_{i}, v_{i}\right) \mathrm{s}$ and the reaction time, every subset $B \subseteq R$ of $m$ clients is equally likely to occur with probability $q=1 /\left(\begin{array}{c}|R| \\ m\end{array}\right)$, and then the expected value for $R$ is

$v_{R}=\sum_{B \subseteq R,|B|=m}\left(\sum_{j \in B} v_{j}\right) q=\frac{m}{|R|} \sum_{j \in R} v_{j}$.

Both cases may be recombined in the formula $v_{R}=$ $\min \{1, m /|R|\} \sum_{j \in R} v_{j}$, with the convention $v_{R}=0$ when $R=\phi$. Finally, assuming that customers act independently, the total expected revenue is

$V(y)=\sum_{R \subseteq C} v_{R} p_{R}(y)=\mathbb{E}\left[\min \left\{1, \frac{m}{\sum_{j \in C} Y_{j}}\right\} \sum_{j \in C} v_{j} Y_{j}\right]$,

where $p_{R}(y)=\prod_{j \in R} y_{j} \prod_{j \notin R}\left(1-y_{j}\right)$ is the probability of observing the respondent set $R$. The optimization problem to be solved is therefore

$$
V^{*}=\max _{0 \leqslant y_{i} \leqslant p_{i}} V(y) .
$$

Although the variables $y_{i}$ are continuous, problem $(P)$ has a discrete nature: Because $V(y)$ is affine with respect to each $y_{i}$, it follows that $(P)$ has an extremal optimal solution with $y_{i} \in\left\{0, p_{i}\right\}$. Indeed, if $y^{*}$ is optimal, the slope corresponding to any "fractional" variable $0<y_{i}^{*}<p_{i}$ must be zero, so that rounding up to $p_{i}$ or down to 0 indifferently does not change the value and yields an extremal solution and a corresponding optimal subset of customers $A^{*}=\left\{i \in C: y_{i}^{*}=p_{i}\right\}$ with no randomization involved. More generally, to any feasible $y$ we may associate an extremal solution $[y]$ with $V([y]) \geqslant V(y)$ by using the following monotone rounding procedure: Consider each fractional variable $0<y_{i}<p_{i}$ sequentially and compare the alternative solutions with $y_{i}=p_{i}$ and $y_{i}=0$, updating $y$ to the best of both. We will denote $A[y]$ the support of this solution, that is, the set of those $i \in C$ with $[y]_{i}>0$.

It follows that, when $C$ is a small set, $(P)$ may be solved by brute force considering all possible subsets $A \subseteq C$ and comparing the values $V_{A}=V\left(y^{A}\right)$ with $y_{i}^{A}=p_{i}$ for $i \in A$ and $y_{i}^{A}=0$ otherwise. This is feasible provided that $V(y)$ can be computed efficiently. The sum in (1) has exponentially many terms and is not well suited for direct computation. An alternative expression may be derived considering the random variables $S=\sum_{j \in C} Y_{j}$ and $S_{i}=\sum_{j \neq i} Y_{j}$, which allows us to write

$$
\begin{aligned}
& V(y)=\sum_{i \in C} v_{i} \pi_{i}(y), \\
& \pi_{i}(y)=\mathbb{E}\left[\min \left\{1, \frac{m}{S}\right\} Y_{i}\right]=y_{i} \mathbb{E}\left[\min \left\{1, \frac{m}{1+S_{i}}\right\}\right],
\end{aligned}
$$

where $\pi_{i}(y)$ represents the probability that customer $i \in C$ gets an item (i.e., he receives an offer, he accepts it, and he is lucky enough to get one of the available items). The distribution of each $S_{i}$ may be computed by convolution in $O\left(n^{2}\right)$ arithmetic operations (see Cormen et al. 2001), so that (2)-(3) provide an effective way to compute $V(y)$ in $O\left(n^{3}\right)$.

\subsection{Conjectures and Counterexamples}

To reduce the effort involved in solving $(P)$ by brute force, one may seek structural properties to limit the search to a smaller range of subsets. Unfortunately, most intuitive conjectures turn out to be false. For instance, because high values $v_{i}$ are preferable it is natural to expect an optimal set of the form $A^{*}=\{1, \ldots, k\}=\left\{i: v_{i} \geqslant \bar{v}\right\}$ for a certain threshold value $\bar{v}$, a common policy in revenue management (Littlewood 1972, Talluri and van Ryzin 2005). In our setting this turns out to be suboptimal, as shown by an elementary example for $m=1$ items and $n=3$ customers with characteristics $p_{1}=p_{2}=0.5, p_{3}=1, v_{1}=2$, $v_{2}=1, v_{3}=0.9$, for which the optimal solution is $A^{*}=$ $\{1,3\}$. Roughly speaking, a customer is valuable only if his contribution to reducing the risk of not selling all the items compensates the losses produced when he displaces a higher valued client. In the example above, the second player is not valuable enough but the third is, despite the fact that $v_{2}>v_{3}$. This occurs because $p_{2}$ is too small while 
$p_{3}$ is large enough, showing that the decision to include a customer depends not only on the values but also the probabilities of all clients.

In general, the highest value customer is always in the optimal set as he cannot displace any higher valued demand. Also using (2) it follows that if $i \in A^{*}$ then $A^{*}$ contains all customers $j$ satisfying $v_{j}>v_{i}$ and $p_{j} \geqslant p_{i}$ simultaneously. Observe that this implies that if $p_{i} \mathrm{~s}$ and $v_{i} \mathrm{~s}$ are positively correlated, then the optimal solution is a threshold strategy, and thus can be found efficiently. However, except for such simple properties, the structure of the optimal set has little to do with the order of the $v_{i} \mathrm{~s}$. As a matter of fact, any set containing the highest value customer can be optimal. More precisely, let $\bar{A} \subseteq \mathbb{Z}_{+}$be any finite set with $1 \in \bar{A}$. Then one can find an instance with $m=1$, in which customers are strictly ordered by decreasing values $v_{1}>v_{2}>\cdots>v_{n}$, and where the optimal solution is exactly $A^{*}=\bar{A}$. To build such an instance, let $C=$ $\{1, \ldots, n\}$ with $n$ to be set later on, and take momentarily $v_{1}=10, p_{1}=0.1$ and $v_{i}=1, p_{i}=\varepsilon$ for $i=2, \ldots, n$. Now, any subset $A$ including customer 1 together with $k$ additional clients has expected revenue

$V_{\varepsilon}(k)=1-\frac{0.9}{(k+1) \varepsilon}\left[(1-\varepsilon)^{k+1}-1+(k+1)(1-\varepsilon)^{k} \varepsilon\right]$.

Considering the monotone change of variables $y=(1-\varepsilon)^{k+1}$, we have $V_{\varepsilon}(k)=1-(0.9 \ln (1-\varepsilon) / \varepsilon) g(y)$, where $g(y)=(y-1) / \ln y+y(\varepsilon /((1-\varepsilon) \ln (1-\varepsilon)))$ is strictly concave and attains its maximum at a unique point $y_{\varepsilon} \in(0,1)$. It turns out that $V_{\varepsilon}(k)$ as a function of the continuous variable $k$ has a unique maximum, which is characterized by the first-order optimality condition. More explicitly, taking $\lambda$ such that $e^{-\lambda}=1-\varepsilon$ and setting $x=k+1$, this optimality condition reads $e^{\lambda x}=\lambda x\left(x\left(e^{\lambda}-1\right)+1\right)+1$. We observe that for any $x>1$, by taking $\lambda=1 / x$ the right-hand side of this equation is larger, while for $\lambda$ big the left-hand side dominates. Hence, there exists $\lambda$ for which the equality holds at $x$. In particular, for $x=|\bar{A}|$ one may find $\varepsilon>0$, such that the maximum of $V_{\varepsilon}(k)$ is attained precisely at $\bar{k}=|\bar{A}|-1$. Now, using this fact and taking $n>\max \{i: i \in \bar{A}\}$, it follows that any subset of $C$ that contains customer 1 and $\bar{k}$ additional customers is optimal. Moreover, if we decrease the probabilities $p_{i}=\varepsilon$ to $p_{i}<\varepsilon$ for all $i \in C \backslash \bar{A}$, we get that the only optimal solution is $\bar{A}$. Finally, we may perturb the values $v_{i}$ so that $v_{1}>v_{2}>\cdots>v_{n}$, with a perturbation small enough to ensure that $\bar{A}$ remains the unique optimal solution.

Given the previous counterexample, one might think that sorting the customers according to an alternative criterion such as expected revenue $p_{i} v_{i}$ could be more appropriate (except for the highest valued customer who is always included in $A^{*}$ ). However, not only can the optimal set differ significantly from any set complying with the order of $p_{i} v_{i} \mathrm{~s}$, but also any such solution may be arbitrarily poor. Indeed, let $\varepsilon>0$ and consider the instance with $C=C_{1} \cup$
$C_{2}=\{1, \ldots, n\}$ where $n$ is sufficiently large. Take $v_{i}=1$, $p_{i}=1$ for $i \in C_{1}$, and $v_{i}=1 / \varepsilon, p_{i}=\varepsilon$ for $i \in C_{2}$, and slightly perturb the values $v_{i}$ so that the customers in $C_{1}$ have slightly higher $p_{i} v_{i}$. Clearly, for large $\left|C_{2}\right|$, the optimal solution has value $V^{*}$ arbitrarily close to $1 / \varepsilon$. On the other hand, if in addition $\left|C_{1}\right| \gg\left|C_{2}\right|$, the best solution complying with the order of $p_{i} v_{i}$ s has value arbitrarily close to 1 .

\section{A Simple Approximation Algorithm}

Although the computational complexity of problem $(P)$ is open, the examples in $\$ 2.2$ suggest that an optimal solution might have a complicated structure. These observations motivate the following question: Can the best $\{1, \ldots, k\}$ strategy be far away from $V^{*}$ ? In the sequel, we answer this question in the negative by providing constant factor approximation algorithms returning solutions of this form. These algorithms run in polynomial time and have an approximation guarantee that improves and becomes asymptotically optimal as the number of items $m$ increases. Because $V(y)$ can be computed in $O\left(n^{3}\right)$, the best threshold solution for the form $A^{*}=\{1, \ldots, k\}$ can be found in $O\left(n^{4}\right)$ arithmetic operations by running the following algorithm.

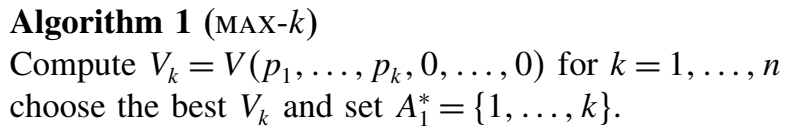

We will measure how far the solution $A_{1}^{*}$ might be from the optimal value by comparing to an even simpler method that requires only two function evaluations with an $O\left(n^{3}\right)$ complexity. Surprisingly, this trivial heuristic computes a threshold-type solution that becomes asymptotically optimal when the number of items $m$ is large.

\section{Algorithm 2 (ADD- $m$ )}

Find the largest $k$ with $p_{1}+\cdots+p_{k} \leqslant m$; Let $\bar{z}=\left(p_{1}, \ldots, p_{k}, \rho, 0, \ldots, 0\right)$ with $\rho=m-\sum_{i=1}^{k} p_{i}$; Set $A_{2}^{*}=A[\bar{z}]$ the support of the monotone rounding of $\bar{z}$.

Note that a randomized variant of this method, which computes $\bar{z}$ and then selects $\{1, \ldots, k\}$ with probability $1-$ $\rho / p_{k+1}$ and $\{1, \ldots, k+1\}$ with probability $\rho / p_{k+1}$, gives a solution whose expected value satisfies all the guarantees we prove for Algorithm 2. Because no monotone rounding is involved, this variant runs in $O(n)$ without ever computing the objective function!

To estimate the quality of the solutions $A_{1}^{*}$ and $A_{2}^{*}$, we consider the following relaxation of $(P)$. We observe from (3) that $\pi_{i}(y) \leqslant y_{i}$ and $\sum_{i} \pi_{i}(y)=\mathbb{E}[\min \{S, m\}] \leqslant m$, so that $V^{*}$ may be bounded from above by the linear program

$$
V^{*} \leqslant \max _{z}\left\{\sum_{i} v_{i} z_{i}: z_{i} \in\left[0, p_{i}\right] ; \sum_{i} z_{i} \leqslant m\right\}=\sum_{i} v_{i} \bar{z}_{i}=\bar{V},
$$

where the optimum $\bar{z}$ is obtained by putting as much weight as possible on higher valued customers, which is precisely the solution computed in ADD- $m$. Clearly, $V(\bar{z}) \leqslant V^{*} \leqslant \bar{V}$ 
and because the set $A_{2}^{*}$ corresponds to the monotone rounding $[\bar{z}]$ we deduce

$V(\bar{z}) \leqslant V_{A_{2}^{*}} \leqslant V_{A_{1}^{*}} \leqslant V^{*} \leqslant \bar{V}$.

A lower bound showing that $V(\bar{z})$ is not too far from the optimal value $V^{*}$ is obtained by letting

$\rho(y)=\mathbb{E}\left[\min \left\{1, \frac{m}{1+S}\right\}\right]$,

so that $\pi_{i}(y) \geqslant y_{i} \rho(y)$. Hence $V(y) \geqslant \rho(y) \sum_{i} v_{i} y_{i}$, from which we get Lemma 1.

LEMMA $1 . V(\bar{z}) \geqslant \rho(\bar{z}) \bar{V}$.

\subsection{The Single Item Case}

A direct consequence of (4) and Lemma 1 is that $\bar{z}$ yields a $1 / 2$-approximate solution if $m=1$.

Proposition 2. If $m=1$, then $V(\bar{z}) \geqslant(1 / 2) V^{*}$ and $A_{1}^{*}, A_{2}^{*}$ are 1/2-approximate solutions for $(P)$.

Proof. It suffices to note that for $\bar{z}$ we have $\mathbb{E}(S)=1$ and then $\rho(\bar{z})=\mathbb{E}(1 /(1+S)) \geqslant 1 /(1+\mathbb{E}(S))=\frac{1}{2}$. Hence $V(\bar{z}) \geqslant \frac{1}{2} \bar{V}$, which combined with (4) gives the desired conclusion.

The bound of $1 / 2$ is tight for $m=1$. For instance, if $v_{1}=1, p_{1}=\varepsilon$ and $v_{2}=0, p_{2}=1-\varepsilon$, the optimal solution is $y^{*}=\left(p_{1}, 0\right)$ with value $V^{*}=\varepsilon$, while the relaxed problem gives $\bar{z}=\left(p_{1}, p_{2}\right)$ with $V(\bar{z})=(1 / 2)\left[\varepsilon+\varepsilon^{2}\right]$. The ratio $V(\bar{z}) / V^{*}$ clearly tends to $1 / 2$ as $\varepsilon \downarrow 0$. Thus our analysis is tight.

\subsection{The Multiple Item Case}

To prove the asymptotic optimality of $\bar{z}$ when $m \rightarrow \infty$, we establish a lower bound for $\rho(\bar{z})$ that converges to 1 . To this end we note that using the Cauchy-Schwartz inequality, we get

$$
\begin{aligned}
\rho(\bar{z}) & =1-\mathbb{E}\left[\frac{1+S-m}{1+S} \mathbb{1}_{\{S \geqslant m\}}\right] \\
& \geqslant 1-\frac{1}{m+1} \mathbb{E}\left[(1+S-m) \mathbb{1}_{\{S \geqslant m\}}\right] \\
& \geqslant 1-\frac{1}{m+1} \sqrt{\mathbb{E}\left[(1+S-m)^{2}\right]} \sqrt{\mathbb{P}(S \geqslant m) .}
\end{aligned}
$$

Because $\mathbb{E}(S-m)=0$ we have $\mathbb{E}\left[(1+S-m)^{2}\right]=1+\bar{\sigma}^{2}$, where $\bar{\sigma}^{2}=\operatorname{Var}(S)=\sum_{i} \bar{z}_{i}\left(1-\bar{z}_{i}\right)$, and then

$\rho(\bar{z}) \geqslant 1-\frac{\sqrt{1+\bar{\sigma}^{2}}}{m+1} \sqrt{\mathbb{P}(S \geqslant m)}$.

Now, $\bar{\sigma}^{2} \leqslant m$ and $\mathbb{P}(S \geqslant m) \leqslant 1$ imply the lower bound $\rho(\bar{z}) \geqslant 1-1 / \sqrt{m+1}$, showing that $V(\bar{z})$ is asymptotically optimal for large $m$. A sharper bound can be obtained using Jogdeo and Samuels (1968, Theorem 3.2), who state that "if the mean number of successes in $n$ independent heterogeneous Bernoulli trials is an integer $m$ then the median is also $m$ " (see Siegel 2001).
Proposition 3. Let $\bar{\sigma}^{2}=\sum_{i} \bar{z}_{i}\left(1-\bar{z}_{i}\right)$. Then $\rho(\bar{z}) \geqslant 1-$ $(1 / \sqrt{m+1}) \sqrt{\frac{1}{2}+1 /(2 \sqrt{m})}$.

Proof. Because $\mathbb{E}(S)=m \in \mathbb{N}$, the median of $S$ is also $m$, that is to say $\mathbb{P}(S>m) \leqslant \frac{1}{2} \leqslant \mathbb{P}(S \geqslant m)$, and therefore $\mathbb{P}(S \geqslant m)=\mathbb{P}(S>m)+\mathbb{P}(S=m) \leqslant 1 / 2+$ $\mathbb{P}(S=m)$. Now, according to Cominetti and Vaisman (2008) and Vaisman (2005) we have $\mathbb{P}(S=m) \leqslant M / \bar{\sigma}$ with $M=\max _{u \geqslant 0} \sqrt{2 u} e^{-2 u} \sum_{k=0}^{\infty}\left(u^{k} / k !\right)^{2}<1 / 2$, so that $\mathbb{P}(S \geqslant m) \leqslant \min \{1,1 / 2+1 /(2 \bar{\sigma})\}$, which plugged into (5) yields

$\rho(\bar{z}) \geqslant 1-\frac{\sqrt{1+\bar{\sigma}^{2}}}{m+1} \min \left\{1, \sqrt{\frac{1}{2}+\frac{1}{2 \bar{\sigma}}}\right\}$.

Because the map $\sigma \mapsto\left(1+\sigma^{2}\right) \min \left\{1, \frac{1}{2}+1 /(2 \sigma)\right\}$ is increasing, the conclusion follows from $\bar{\sigma} \leqslant \sqrt{m}$.

Proposition 3 implies that the solutions $A_{1}^{*}$ and $A_{2}^{*}$ are asymptotically optimal with rate of convergence of order $1 / \sqrt{m}$. This rate cannot be improved because of the central limit theorem. Indeed, consider a large instance of $n$ identical clients with $v_{i}=1$ and $p_{i}=p$. The optimal solution for any number of items $m$ is to include all customers $A^{*}=C$, with optimal value $V^{*} \sim m$ for $n$ large enough. Now suppose that $p=m / k$ with $k$ integer so that $A_{2}^{*}=\{1, \ldots, k\}$ which according to (1) gives a value

$$
\begin{aligned}
V_{A_{2}^{*}} & =\mathbb{E}[\min \{S, m\}] \\
& =m+\mathbb{E}\left[\min \left\{\frac{S-m}{\sqrt{m(1-p)}}, 0\right\}\right] \sqrt{m(1-p)},
\end{aligned}
$$

where $S$ has binomial distribution $B(k, p)$ with $\mathbb{E}(S)=m$ and $\operatorname{Var}(S)=m(1-p)$. If we now consider a case with $p$ fixed and $m, k$ large, the central limit theorem implies that $V_{A_{2}^{*}} \sim m[1-\sqrt{(1-p) /(2 \pi m)}]$, which underestimates the optimal value by a factor of the order $1 / \sqrt{m}$.

\subsection{An Alternative Approach for the Single Item Case}

Both solutions $A_{1}^{*}$ and $A_{2}^{*}$ provide a lower bound for the optimal value $V^{*}$. We proved in Proposition 2 that for $m=1$ the value $V_{A_{2}^{*}}$ is at least $(1 / 2) \bar{V}$ so that $V^{*} \in[(1 / 2) \bar{V}, \bar{V}]$. An improved lower bound (and a corresponding approximate solution) can be obtained using (3) and noting that $\pi_{i}(y)=y_{i} \mathbb{E}\left[1 /\left(1+S_{i}\right)\right] \geqslant$ $y_{i} /\left(1+\mathbb{E}\left(S_{i}\right)\right)=y_{i} /\left(1+\sum_{j \neq i} y_{j}\right) \geqslant y_{i} /\left(1+\sum_{j \in C} y_{j}\right)$. Therefore, $V^{*} \geqslant \tilde{V}$, with $\tilde{V}=\max _{0 \leqslant y_{i} \leqslant p_{i}}\left(\sum_{i \in C} v_{i} y_{i}\right) /$ $\left(1+\sum_{i \in C} y_{i}\right)$. The latter is closely related to the common lines problem in transit network optimization (Chriqui and Robillard 1975) and can be easily solved by a linear time algorithm.

Algorithm 3 (MAX-AVG)

Find $k$ with $\left[v_{1} p_{1}+\cdots+v_{k} p_{k}\right] /\left[1+p_{1}+\cdots+p_{k}\right]$ maximal set $A_{3}^{*}=\{1, \ldots, k\}$. 
The set found by this algorithm satisfies $\tilde{V} \leqslant V_{A_{3}^{*}} \leqslant V^{*}$. Note that for each $z$ feasible in the relaxation $\bar{V}$ we have

$\sum_{i \in C} v_{i} z_{i} \leqslant 2 \frac{\sum_{i \in C} v_{i} z_{i}}{1+\sum_{i \in C} z_{i}} \leqslant 2 \widetilde{V}$,

from which we get $(1 / 2) \bar{V} \leqslant \tilde{V}$ so that $\tilde{V}$ is a sharper lower bound and then $V^{*} \in[\tilde{V}, \bar{V}]$. It follows that $A_{3}^{*}$ is a 1/2-approximate solution for $(P)$ as well.

\section{A Sharper Relaxation}

We now derive an improved LP relaxation of $(P)$, which follows from a nonlinear nonconvex formulation, and use it to obtain a 2/3-approximation algorithm for the single item case. Using the expression $V(y)=\sum_{R \subseteq C} v_{R} p_{R}(y)$ given in (1) and introducing new variables $x_{R}=p_{R}(y)$, we have

$$
\begin{aligned}
V^{*}= & \max \sum_{R \subseteq C} v_{R} x_{R} \\
& 0 \leqslant y_{i} \leqslant p_{i} \quad \text { for all } i \in C \\
& x_{R}=\prod_{j \in R} y_{j} \prod_{j \notin R}\left(1-y_{j}\right) \quad \text { for all } R \subseteq C .
\end{aligned}
$$

The $x_{R} \mathrm{~s}$ are equivalently defined by the constraints $x_{R}=$ $x_{R \backslash\{i\}}\left(y_{i} /\left(1-y_{i}\right)\right)$ for all $i \in R$ together with $\sum_{R \subseteq C} x_{R}=1$, so that the problem becomes

$$
\begin{aligned}
V^{*}= & \max \sum_{R \subseteq C} v_{R} x_{R} \\
& 0 \leqslant y_{i} \leqslant p_{i} \quad \text { for all } i \in C \\
& x_{R}=x_{R \backslash\{i\}} \frac{y_{i}}{1-y_{i}} \quad \text { for all } R \subseteq C, i \in R \\
& \sum_{R \subseteq C} x_{R}=1,
\end{aligned}
$$

which may then be relaxed into the following linear program:

$$
\begin{aligned}
V^{*} \leqslant V_{L P}= & \max \sum_{R \subseteq C} v_{R} x_{R} \\
& 0 \leqslant x_{R} \leqslant x_{R \backslash\{i\}} \frac{p_{i}}{1-p_{i}} \quad \text { for all } R \subseteq C, i \in R \\
& \sum_{R \subseteq C} x_{R}=1 .
\end{aligned}
$$

Although there are instances for which $V^{*}<V_{L P}$, the following property of extremal solutions for $V_{L P}$ provides intuition on the structure of the above linear program.

Lemma 4. Let $x$ be a basic solution for $V_{L P}$. Then for all $R \neq \phi$ such that $x_{R}>0$, we have

$x_{R}=x_{\phi} \prod_{i \in R} \frac{p_{i}}{1-p_{i}}$.

Proof. The inequalities $x_{R} \leqslant x_{R \backslash\{i\}}\left(p_{i} /\left(1-p_{i}\right)\right)$ inductively imply $x_{R} \leqslant x_{\phi} \prod_{i \in R}\left(p_{i} /\left(1-p_{i}\right)\right)$ and then, because the sum of the $x_{R} \mathrm{~s}$ is 1 , it follows that $x_{\phi}>0$.
Let us build a graph $G=(N, E)$ with one vertex $n_{R} \in N$ for each $R \subseteq C$ with $x_{R}>0$, and an edge $\left(n_{R}, n_{R \backslash\{i\}}\right) \in$ $E$ iff $i \in R$ and $x_{R}=x_{R \backslash\{i\}}\left(p_{i} /\left(1-p_{i}\right)\right)$. We claim that $G$ is connected, from which (6) follows at once. If this was not the case, we could find a nontrivial partition of $N$ into $L \cup U$ with $n_{\phi} \in L$, such that $x_{R}<x_{R \backslash\{i\}}\left(p_{i} /\left(1-p_{i}\right)\right)$ when $n_{R} \in U$ and $n_{R \backslash\{i\}} \in L$. Let $\varepsilon, \delta>0$ be such that $\varepsilon \sum_{R \in U} x_{R}=\delta \sum_{R \in L} x_{R}$, and small enough so that $\bar{x}$ and $\tilde{x}$ defined as

$\bar{x}_{R}=\left\{\begin{array}{ll}x_{R}(1-\varepsilon), & \text { for all } R \in U \\ x_{R}(1+\delta), & \text { for all } R \in L,\end{array}\right.$ and
$\tilde{x}_{R}=\left\{\begin{array}{ll}x_{R}(1+\varepsilon), & \text { for all } R \in U \\ x_{R}(1-\delta), & \text { for all } R \in L,\end{array}\right.$ and

are feasible for LP. It follows immediately that $x=$ $(1 / 2) \bar{x}+(1 / 2) \tilde{x}$, and thus $x$ is not an extreme point. This contradiction shows that $G$ is connected, which completes the proof.

Although $V_{L P}$ seems to be a fairly tight upper bound on $V^{*}$, it might be hard to compute since it has exponentially many variables and constraints. We may, however, derive a weaker relaxation for the single item case, which yields a 2/3-approximation algorithm. Let us rewrite the LP as

$$
\begin{aligned}
V_{L P}= & \max \sum_{i \in C} v_{i} \sum_{R \ni i} \frac{x_{R}}{|R|} \\
& 0 \leqslant x_{R} \leqslant p_{i}\left(x_{R \backslash\{i\}}+x_{R}\right) \quad \text { for all } R \subseteq C, i \in R \\
& \sum_{R \subseteq C} x_{R}=1 .
\end{aligned}
$$

Letting $z_{i}=\sum_{R \ni i}\left(x_{R} /|R|\right)$, we get $\sum_{i \in C} z_{i}=1-x_{\phi}$, while the inequalities $x_{R} \leqslant p_{i}\left(x_{R \backslash\{i\}}+x_{R}\right)$ yield

$$
\begin{aligned}
z_{i} \leqslant & \sum_{R \ni i} \frac{p_{i}\left(x_{R \backslash\{i\}}+x_{R}\right)}{|R|} \leqslant p_{i}\left(x_{\phi}+x_{\{i\}}\right) \\
& +\frac{p_{i}}{2} \sum_{R \ni i,|R| \geqslant 2}\left(x_{R \backslash\{i\}}+x_{R}\right) .
\end{aligned}
$$

Because $\sum_{R} x_{R}=1$ the last sum is equal to $1-x_{\phi}-x_{\{i\}}$, so that $z_{i} \leqslant\left(p_{i} / 2\right)\left(x_{\phi}+x_{\{i\}}+1\right) \leqslant\left(p_{i} / 2\right)\left[x_{\phi} /\left(1-p_{i}\right)+1\right]$. Combining these inequalities and renaming the variable $x_{\phi}$ as $x$, we get

$$
\begin{aligned}
& V_{L P} \leqslant V_{L P_{2}}=\max \sum_{i \in C} v_{i} z_{i} \\
& z_{i} \leqslant \frac{p_{i}}{2}\left[\frac{x}{1-p_{i}}+1\right] \text { for all } i \in C \\
& \sum_{i \in C} z_{i}=1-x \\
& z_{i}, x \geqslant 0,
\end{aligned}
$$

from which we derive the following alternative approximation algorithm. 


\section{Algorithm 4 (LP-RELAX)}

Find a basic optimal solution $\left(z^{*}, x^{*}\right)$ for $V_{L P_{2}}$ and let $y_{i}=$ $2 z_{i}^{*} /\left(x^{*} /\left(1-p_{i}\right)+1\right)$

Set $A_{4}^{*}=A[y]$ the support of the monotone rounding of $y$.

Note that the solution given by this algorithm is also of threshold type $A_{4}^{*}=\{1, \ldots, k\}$. Indeed, once the optimal $x^{*}$ is known, the optimal values $z_{i}^{*}$ are obtained by putting as much weight as possible on high-value customers, saturating the constraints.

Proposition 5. When $m=1$, we have $V_{A_{4}^{*}} \geqslant \frac{2}{3} V^{*}$.

Proof. Because $V_{A_{4}^{*}} \geqslant V(y)$ and $V_{L P_{2}} \geqslant V^{*}$, it suffices to show $V(y) \geqslant \frac{2}{3} V_{L P_{2}}$. To this end we write

$V(y)=\sum_{i \in C} v_{i} y_{i} \mathbb{E}\left(\frac{1}{1+\sum_{j \neq i} Y_{j}}\right)=\sum_{i \in C} v_{i} z_{i}^{*} \gamma_{i}$,

with $\gamma_{i}=2 \mathbb{E}\left(1 /\left(1+\sum_{j \neq i} Y_{j}\right)\right) /\left(1+x^{*} /\left(1-p_{i}\right)\right)$. Let us prove that $\gamma_{i} \geqslant 2 / 3$ whenever $z_{i}^{*}>0$. Indeed, Jensen's inequality implies $\gamma_{i} \geqslant 2 /\left[\left(1+\sum_{j \neq i} y_{j}\right)\left(1+x^{*} /\left(1-p_{i}\right)\right)\right]$, and because

$\sum_{j \neq i} y_{j}=\sum_{j \neq i} \frac{2 z_{j}^{*}}{1+x^{*} /\left(1-p_{j}\right)} \leqslant \frac{2}{1+x^{*}} \sum_{j \neq i} z_{j}^{*}=\frac{2\left(1-x^{*}-z_{i}^{*}\right)}{1+x^{*}}$,

we deduce

$\gamma_{i} \geqslant \frac{2\left(1+x^{*}\right)}{\left(3-x^{*}-2 z_{i}^{*}\right)\left(1+x^{*} /\left(1-p_{i}\right)\right)}$.

When $x^{*}=0$, the inequality $\gamma_{i} \geqslant 2 / 3$ follows at once. For the case $x^{*}>0$ we note that because $\left(z^{*}, x^{*}\right)$ is a basic solution, exactly one of the two inequalities involving each $z_{i}^{*}$ must be tight so that $z_{i}^{*}>0$ implies $z_{i}^{*}=\left(p_{i} / 2\right)\left(1+x^{*} /\right.$ $\left.\left(1-p_{i}\right)\right)$, which plugged into the previous bound yields

$\gamma_{i} \geqslant \frac{2\left(1+x^{*}\right)}{\left(3-p_{i}-x^{*} /\left(1-p_{i}\right)\right)\left(1+x^{*} /\left(1-p_{i}\right)\right)}$.

The conclusion follows because the latter is at least $2 / 3$ for all $x^{*} \in[0,1]$.

Note that if we have three clients with characteristics $p_{1}=\varepsilon, p_{2}=p_{3}=1-\varepsilon / 2, v_{1}=1, v_{2}=v_{3}=\varepsilon /(2-\varepsilon)$, we have that $V_{L P_{2}}=V^{*}=\varepsilon$ and $A_{4}^{*}=\{1,2,3\}$, whose value approaches $5 \varepsilon / 6$ for $\varepsilon$ small. Thus our algorithm is not better than a 5/6-approximation. On the other hand, our analysis in the last proposition is tight. Indeed, the clientby-client bound $\gamma_{i}$ that we use to prove the result can actually achieve the value $2 / 3$. To see this note that in the same three-client instance above the value $z_{1}$ in $L P_{2}$ is $\varepsilon / 2$, while in $V\left(A_{4}^{*}\right)$ the coefficient accompanying $v_{1}$ approaches $\varepsilon / 3$.

\section{A PTAS for the Single Item Case}

In this section we provide polynomial time approximation scheme (PTAS) for the single item case, namely, given $\varepsilon>0$ we show how to compute (in time which is polynomial for fixed $\varepsilon$ ) a strategy that yields a value within a factor $(1+\varepsilon)$ from the optimum. Recall that after introducing $(P)$ in $\S 2$ we noted that this problem has an equivalent combinatorial version, namely

$\max _{A \subseteq N} V_{A}:=\sum_{R \subseteq A} v_{R} p_{R}$,

where $p_{R}=\prod_{i \in R} p_{i} \cdot \prod_{i \in A \backslash R}\left(1-p_{i}\right)$. We say a client $i \in$ $N$ is large if $p_{i}>\varepsilon$ and small otherwise. We will show that the following algorithm computes an $(1+O(\sqrt{\varepsilon}))$ approximate solution:

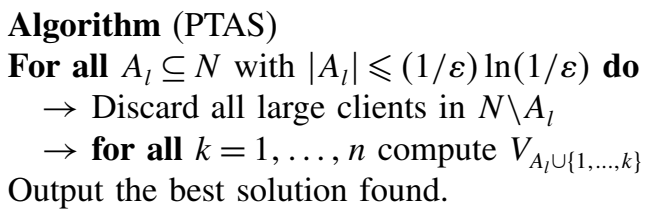

The running time of the algorithm can be estimated as follows. We try at most $\left(\begin{array}{c}n \\ (1 / \varepsilon) \ln (1 / \varepsilon)\end{array}\right)$ subsets $A_{l}$ and, for each one, we evaluate $V_{A_{1} \cup\{1, \ldots, k\}}$ for $k=1, \ldots, n$. Because each evaluation takes $O\left(n^{3}\right)$, the overall running time of the algorithm is $O\left(n^{4+\ln (1 / \varepsilon) / \varepsilon}\right)$, which is polynomial for fixed $\varepsilon$.

To establish the $(1+O(\sqrt{\varepsilon}))$ approximation guarantee we proceed as follows. First, we show that restricting the optimization in (7) to strategies with at most $(1 / \varepsilon) \ln (1 / \varepsilon)$ large clients, accounts for a loss factor of at most $(1+O(\varepsilon))$. Therefore, it suffices to check all strategies $A$ that contain a subset of large clients $A_{l}$ of size at most $(1 / \varepsilon) \ln (1 / \varepsilon)$. Second, for the small clients we will show that the best threshold strategy is near optimal. This is precisely what the algorithm does.

\subsection{Restricting the Optimal Strategy}

We show that there is a near-optimal solution for $(P)$ whose sum of probabilities is not too large.

Lemma 6. For small $\varepsilon>0$ there is $A \subseteq N$ with $\sum_{i \in A} p_{i} \leqslant$ $\ln (1 / \varepsilon)$ such that $V^{*} \leqslant(1+3 \varepsilon) V_{A}$.

Proof. Take $A^{*}$ optimal for $(P)$ and assume without loss of generality that $A^{*}=\{1, \ldots, n\}$. If $\sum_{i \in A^{*}} p_{i} \leqslant \ln (1 / \varepsilon)$ just take $A=A^{*}$. Otherwise, let $A:=\{1, \ldots, k\} \subseteq A^{*}$ with $k$ the largest integer satisfying $\sum_{i=1}^{k} p_{i} \leqslant \ln (1 / \varepsilon)$. The probability that no customer in $A$ accepts can be bounded as

$q:=\mathbb{P}[$ no customer in $A$ accepts $]$

$$
=\prod_{i \in A}\left(1-p_{i}\right) \leqslant e^{-\sum_{i=1}^{k} p_{i}} \leqslant e^{-(\ln (1 / \varepsilon)-1)}=e \varepsilon,
$$

where the first inequality follows from $1-x \leqslant e^{-x}$ and the second from the definition of $k$. 
When no customer in $A$ accepts the strategy, $A$ gets zero revenue while $A^{*}$ gets at most $v_{k}$. On the other hand, if some customers in $A$ accept, then strategy $A$ gets more revenue than $A^{*}$, and of course revenue at least $v_{k}$. Thus, for $\varepsilon \leqslant(3-e) / 3 e$, we have $V_{A^{*}}-V_{A} \leqslant v_{k} \leqslant q /(1-q) V_{A} \leqslant$ $3 \varepsilon V_{A}$.

According to this lemma, we may restrict to strategies $A$ such that $\sum_{i \in A} p_{i} \leqslant \ln (1 / \varepsilon)$, which accounts for a loss of at most a factor $1+O(\varepsilon)$.

\subsection{Modifying the Instance}

The next step is to consider a modified instance that is easier to solve and from which we recover an approximate solution for the original problem $(P)$. The idea is to split each small client $p_{i} \leqslant \varepsilon$ into several clones with an even smaller probability $\delta$, and to show that this does not affect too much the expected revenue. More precisely, given an instance $I=\left\{\left(v_{1}, p_{1}\right), \ldots,\left(v_{n}, p_{n}\right)\right\}$ with set of clients $N$ and a small $\delta>0$, we build an instance $I^{\prime}$ that contains all large customers in $I$; and also for each small customer $i \in I$, we add to $I^{\prime}$ a number $q_{i}$ of clones with value $v_{i}$ and probability $\delta$. Here $q_{i}$ is chosen to be the largest integer satisfying

$1-p_{i} \leqslant(1-\delta)^{q_{i}}$

Observe that the quantity $\tilde{p}_{i}:=1-(1-\delta)^{q_{i}}$ can be made arbitrarily close to $p_{i}$ by taking $\delta$ small enough. For convenience, we denote $\tilde{I}$ the perturbed instance of $I$ in which each small $p_{i}$ is changed into $\tilde{p}_{i}$. By continuity, for any $A \subset N$, the value $V_{A}$ in both instances $I$ and $\tilde{I}$ are arbitrarily close. Hence, by choosing $\delta$ small enough we can make the optimal values of $I$ and $\tilde{I}$ lie within a factor arbitrarily close to 1 .

Let $N^{\prime}$ denote the set of clients in $I^{\prime}$ and let $\sigma: N^{\prime} \rightarrow N$ so that for each $i \in N$ the set $\sigma^{-1}(i)$ denotes the corresponding clones in $N^{\prime}$ (just one if client $i$ is large). We claim that $I^{\prime}$ and $\tilde{I}$ have very similar optimal values. Roughly speaking, we interpret the value of a strategy $A$ in $I$ as the value under a modified random process in which a client $i \in A$ accepts if and only if at least one clone in $\sigma^{-1}(i)$ accepts in $I^{\prime}$. As usual, for every $j \in N^{\prime}$ we let $Y_{j}^{\prime}$ denote a Bernoulli random variable with $\mathbb{P}\left(Y_{j}^{\prime}=1\right)$ equal to $p_{j}$ if $j$ is a large, and equal to $\delta$ for all clones $j$ of small customers. We will also denote $Y_{i}=\max \left\{Y_{j}^{\prime}: j \in \sigma^{-1}(i)\right\}$ for each $i \in N$ and we observe that these $Y_{i}$ s are independent Bernoulli's of parameter $p_{i}$ for $i \in N$ large and $\tilde{p}_{i}$ for $i \in N$ small. Using these variables the value $V_{A^{\prime}}$ of a strategy $A^{\prime} \subset N^{\prime}$ in the instance $I^{\prime}$ can be expressed as

$V_{A^{\prime}}=\mathbb{E}\left[\sum_{j \in A^{\prime}} v_{j} Y_{j}^{\prime} / \sum_{j \in A^{\prime}} Y_{j}^{\prime}\right]$,

while the value $\tilde{V}_{A}$ of a strategy $A \subset N$ in the instance $\tilde{I}$ is $\tilde{V}_{A}=\mathbb{E}\left[\sum_{i \in A} v_{i} Y_{i} / \sum_{i \in A} Y_{i}\right]$.
We will show that both $A$ and $A^{\prime}=\sigma^{-1}(A)$ yield the same revenue with high probability. To this end we note that the probability that all customers in $\sigma^{-1}(i)$ reject the offer in $I^{\prime}$ is

$\mathbb{P}\left[\sigma^{-1}(i)\right.$ reject $]=(1-\delta)^{q_{i}}=1-\tilde{p}_{i}$,

while the the next lemma shows that the probability that more than one clone in $\sigma^{-1}(i)$ accepts simultaneously is very small.

LeMmA 7. Let $R^{\prime}$ be the respondent customers in $A^{\prime}=$ $\sigma^{-1}(A)$. If $\varepsilon<1 / 2$, then for each small client $i \in N$ we have $\mathbb{P}\left[\left|\sigma^{-1}(i) \cap R^{\prime}\right| \geqslant 2\right] \leqslant 2 \tilde{p}_{i}^{2} \leqslant 2 p_{i}^{2}$.

Proof. We observe that

$$
\begin{aligned}
& \mathbb{P}\left[\left|\sigma^{-1}(i) \cap R^{\prime}\right| \geqslant 2\right]+\mathbb{P}\left[\left|\sigma^{-1}(i) \cap R^{\prime}\right| \geqslant 1\right] \\
& \quad=2 \mathbb{P}\left[\left|\sigma^{-1}(i) \cap R^{\prime}\right| \geqslant 2\right]+\mathbb{P}\left[\left|\sigma^{-1}(i) \cap R^{\prime}\right|=1\right] \\
& \quad \leqslant \mathbb{E}\left[\left|\sigma^{-1}(i) \cap R^{\prime}\right|\right]=q_{i} \delta \leqslant \ln \frac{1}{1-\tilde{p}_{i}} \leqslant \frac{\tilde{p}_{i}}{1-\tilde{p}_{i}}
\end{aligned}
$$

where the last inequalities follow by taking logarithm in (9). Now, using (9) once again we have

$\mathbb{P}\left[\left|\sigma^{-1}(i) \cap R^{\prime}\right| \geqslant 1\right]=1-\mathbb{P}\left[\sigma^{-1}(i)\right.$ reject $]=\tilde{p}_{i}$,

which combined to the previous estimate yields $\mathbb{P}\left[\left|\sigma^{-1}(i) \cap R^{\prime}\right| \geqslant 2\right] \leqslant \tilde{p}_{i} /\left(1-\tilde{p}_{i}\right)-\tilde{p}_{i} \leqslant 2 \tilde{p}_{i}^{2}$.

We will say that a (random) respondent set $R^{\prime}$ for the instance $I^{\prime}$ is good if $\left|\sigma^{-1}(i) \cap R^{\prime}\right| \leqslant 1$ for all small clients $i \in N$. Otherwise, we will say that $R^{\prime}$ is bad.

Lemma 8. Let $A \subseteq N$ with $\sum_{i \in A} \tilde{p}_{i} \leqslant \ln (1 / \varepsilon)$ and $A^{\prime}=$ $\sigma^{-1}(A)$. Then $V_{A^{\prime}} \geqslant(1+O(\sqrt{\varepsilon})) \tilde{V}_{A^{\prime}}$.

Proof. First notice that $\tilde{V}_{A} \leqslant(1+O(\sqrt{\varepsilon}))$. $\sum_{R:|R| \leqslant 1 / \sqrt{\varepsilon}} v_{R} \tilde{p}_{R}$. Indeed,

$$
\begin{aligned}
\tilde{V}_{A} & =\sum_{i \in A} v_{i}\left(\sum_{R \ni i ;|R| \leqslant 1 / \sqrt{\varepsilon}} \frac{\tilde{p}_{R}}{|R|}+\sum_{R \ni i ;|R|>1 / \sqrt{\varepsilon}} \frac{\tilde{p}_{R}}{|R|}\right) \\
& \leqslant \sum_{i \in A} v_{i}\left(\sum_{R \ni i ;|R| \leqslant 1 / \sqrt{\varepsilon}} \frac{\tilde{p}_{R}}{|R|}+\sum_{R \ni i} \tilde{p}_{R} \sqrt{\varepsilon}\right) \\
& \leqslant \sum_{i \in A} v_{i} \sum_{R \ni i ;|R| \leqslant 1 / \sqrt{\varepsilon}} \frac{\tilde{p}_{R}}{|R|}+\sum_{i \in A} v_{i} \tilde{p}_{i} \sqrt{\varepsilon} \\
& \leqslant \sum_{R:|R| \leqslant 1 / \sqrt{\varepsilon}} v_{R} \tilde{p}_{R}+(1-\ln \varepsilon) \sqrt{\varepsilon} \tilde{V}_{A},
\end{aligned}
$$

where the last inequality comes from

$$
\begin{aligned}
\tilde{V}_{A} & =\sum_{i \in A} v_{i} \tilde{p}_{i} \mathbb{E}\left(\frac{1}{1+S_{i}}\right) \\
& \geqslant \sum_{i \in A} v_{i} \tilde{p}_{i} \frac{1}{1+\sum_{i \in A} \tilde{p}_{i}} \geqslant \sum_{i \in A} v_{i} \tilde{p}_{i} \frac{1}{1-\ln \varepsilon} .
\end{aligned}
$$


On the other hand, $V_{A^{\prime}}$ can be bounded as follows:

$$
\begin{aligned}
V_{A^{\prime}} & =\sum_{R \subseteq A} \sum_{R^{\prime}: \sigma\left(R^{\prime}\right)=R} v_{R^{\prime}} p_{R^{\prime}} \\
& \geqslant \sum_{R \subseteq A ;|R| \leqslant 1 / \sqrt{\varepsilon}}\left(\sum_{R^{\prime} \text { good: } \sigma\left(R^{\prime}\right)=R} v_{R^{\prime}} p_{R^{\prime}}\right) .
\end{aligned}
$$

Now, for all $R^{\prime}$ good we have $v_{R^{\prime}}=v_{R}$, while $\sum_{R^{\prime} \text { good: } \sigma\left(R^{\prime}\right)=R} p_{R^{\prime}}$ is the probability that a random $R^{\prime}$ is good and $\sigma\left(R^{\prime}\right)=R$; that is to say

$$
\begin{array}{r}
\prod_{i \notin R} \mathbb{P}\left[\left|\sigma^{-1}(i) \cap R^{\prime}\right|=0\right] \prod_{i \in R} \mathbb{P}\left[\left|\sigma^{-1}(i) \cap R^{\prime}\right|=1\right] \\
\geqslant \prod_{i \notin R}\left(1-\tilde{p}_{i}\right) \prod_{i \in R}\left(\tilde{p}_{i}-2 \tilde{p}_{i}^{2}\right)=\tilde{p}_{R} \prod_{i \in R}\left(1-2 \tilde{p}_{i}\right),
\end{array}
$$

where the inequality follows from Lemma 7. Plugging the latter into (10) we get

$$
\begin{aligned}
V_{A^{\prime}} & \geqslant \sum_{R \subseteq A ;|R| \leqslant 1 / \sqrt{\varepsilon}} v_{R} \tilde{p}_{R} \prod_{i \in R}\left(1-2 p_{i}\right) \\
& \geqslant \sum_{R \subseteq A ;|R| \leqslant 1 / \sqrt{\varepsilon}} v_{R} \tilde{p}_{R}(1-2 \varepsilon)^{1 / \sqrt{\varepsilon}} .
\end{aligned}
$$

The lemma follows by combining this bound with that obtained for $\tilde{V}_{A}$.

Lemma 9. Let $A \subseteq N$ and set $A^{\prime}=\sigma^{-1}(A)$. Then $V_{A^{\prime}} \leqslant$ $(1+O(\varepsilon)) \tilde{V}_{A}$.

Proof. Note that from the definition of $Y_{i}=\max \left\{Y_{j}^{\prime}: j \in\right.$ $\left.\sigma^{-1}(i)\right\} \leqslant \sum_{j \in \sigma^{-1}(i)} Y_{j}^{\prime}$. Then

$$
\begin{aligned}
V_{A^{\prime}} & =\sum_{i \in A} \sum_{j \in \sigma^{-1}(i)} v_{j} p_{j} \mathbb{E}\left(\frac{1}{1+\sum_{k \neq j} Y_{k}^{\prime}}\right) \\
& \leqslant \sum_{i \in A} v_{i} \sum_{j \in \sigma^{-1}(i)} p_{j} \mathbb{E}\left(\frac{1}{1+\sum_{l \in A, l \neq i} \sum_{k \in \sigma^{-1}(l)} Y_{k}^{\prime}}\right) \\
& \leqslant \sum_{i \in A} v_{i} \mathbb{E}\left(\frac{1}{1+\sum_{l \neq i} Y_{l}}\right) \sum_{j \in \sigma^{-1}(i)} p_{j} .
\end{aligned}
$$

To conclude, observe that if $i$ is big, then $\sigma^{-1}(i)$ is a singleton and $\sum_{j \in \sigma^{-1}(i)} p_{j}=p_{i}$, while for $i$ small we have $\sum_{j \in \sigma^{-1}(i)} p_{j}=\delta q_{i} \leqslant(1+O(\varepsilon)) \tilde{p}_{i}$.

\subsection{Proof of Approximation Guarantee}

THEOREM 1. For small $\varepsilon>0$ our algorithm computes a $(1+O(\sqrt{\varepsilon}))$-approximate solution in polynomial time.

Proof. Let $V^{\prime}$ be the optimal value of instance $I^{\prime}$. Using Lemma 6 there is $A^{\prime}$ with $\sum_{i \in A^{\prime}} p_{i} \leqslant \ln (1 / \varepsilon)$ whose value is within a factor $(1+O(\varepsilon))$ of $V^{\prime}$. Let $A_{l}$ denote the set of large customers in $A^{\prime}$ so that we necessarily have $\left|A_{l}\right| \leqslant(1 / \varepsilon) \ln (1 / \varepsilon)$. Now, let also $A_{s}$ be the set of small customers in $A^{\prime}$, so that $A_{l} \cup A_{s}=A^{\prime}$. Note that we can assume without loss of generality that $A_{s}$ contains all clones of the highest valued small clients, except eventually for a threshold small client $i^{*}$ for which only a strict subset of its clones $\sigma^{-1}\left(i^{*}\right)$ is considered. Indeed, because all small clients have the same probability $\delta$, if $j \notin A_{s}$ and $k \in A_{s}$ are two small clients with $v_{j}>v_{k}$, then exchanging $j$ and $k$ in $A_{s}$ increases the value of $A^{\prime}$.

We observe that strategy $A=\sigma\left(A^{\prime}\right) \backslash\left\{i^{*}\right\}$ is of the form considered in the algorithm, so that denoting $A^{*}$ the best strategy found by the algorithm, we have

$V_{A} \leqslant V_{A^{*}} \leqslant V^{*}$

Now, consider the strategy $A^{\prime \prime}=\sigma^{-1}(A)$. The only difference between $A^{\prime \prime}$ and $A^{\prime}$ is that $A^{\prime \prime}$ does not contain any clone in $\sigma^{-1}\left(i^{*}\right)$. Thus the value of $A^{\prime \prime}$ is smaller than that of $A^{\prime}$ only if no customer in $A^{\prime \prime}$ accepts and some customer in $A^{\prime}$ does, in which case the difference is exactly $v_{i^{*}}$. This event occurs with probability at most $\varepsilon$, from where we get $V_{A^{\prime}} \leqslant V_{A^{\prime \prime}}+\varepsilon v_{i^{*}}$. Moreover, if $A^{\prime \prime} \neq A^{\prime}$, then $V_{A^{\prime}} \geqslant$ $v_{i^{*}}$, as otherwise including one more clone $j$ of customer $i^{*}$ to $A^{\prime}$ would lead to a strategy whose value is at least $(1-\delta) V_{A^{\prime}}+\delta v_{i^{*}}$. This implies $(1-\varepsilon) V_{A^{\prime}} \leqslant V_{A^{\prime \prime}}$, which combined with Lemmas 8 and 9 yields

$$
\begin{aligned}
\tilde{V}_{A} & =(1+O(\sqrt{\varepsilon})) V_{A^{\prime \prime}} \\
& \geqslant(1+O(\sqrt{\varepsilon})) V_{A^{\prime}}=(1+O(\sqrt{\varepsilon})) V^{\prime} .
\end{aligned}
$$

Clearly, by a continuity argument, we can fix a priori $\delta$ small enough so that the $\tilde{p}_{i}$ s are all sufficiently close to the $p_{i} \mathrm{~s}$ in order to guarantee $V_{B}=(1+O(\varepsilon)) \widetilde{V}_{B}$ for all $B \subseteq N$. In particular, such a $\delta$ guarantees that $V_{A}=(1+O(\varepsilon)) \tilde{V}_{A}$, which combined with (11) and (12) leads to

$V_{A^{*}} \geqslant V_{A} \geqslant(1+O(\sqrt{\varepsilon})) V^{\prime}$.

Now, by Lemma 6 there is a set $\bar{A} \subseteq N$, whose sum of probabilities is at most $\ln (1 / \varepsilon)$, such that $V^{*} \leqslant(1+$ $O(\varepsilon)) V_{\bar{A}}$. Again, the choice of $\delta$ implies $V_{\bar{A}} \leqslant(1+$ $O(\varepsilon)) \tilde{V}_{\bar{A}}$, while Lemma 8 gives

$\tilde{V}_{\bar{A}} \leqslant(1+O(\sqrt{\varepsilon})) V_{\sigma^{-1}(\bar{A})} \leqslant(1+O(\sqrt{\varepsilon})) V^{\prime}$,

so that $V^{*} \leqslant(1+O(\sqrt{\varepsilon})) V^{\prime}$. The latter together with (13) concludes the proof.

\section{Final Remarks}

Throughout the paper we have assumed that customers are selected according to a first-come-first-served discipline. We have argued that this policy is reasonable in our short-term setting, because it only assumes that customers response times are independent and identically distributed, and moreover, this seems to be the only alternative because it may be too difficult, or too late, to discriminate among clients. In addition, note that our approximation results concerning threshold policies in $\$ 3$, use as a comparison 
benchmark (upper bound) $\bar{V}$, the solution of a linear program that is also an upper bound for the revenue of any rationing policy, so that the potential gain of using a more sophisticated policy is still at most $O(1 / \sqrt{m})$. In particular, for 10 items, our simple policies are guaranteed to obtain at least $80 \%$ of the revenue of any rationing policy, and this fraction grows to 1 as the number of items to be sold grows.

Let us now comment on further research directions. First, a natural extension, related to stochastic knapsack (Dean et al. 2008), is the following. As before, customers accept the offer with probabilities $p_{i}$ and contribute a value $v_{i}$ if they buy an item. The difference is that customer $i \in C$ demands $s_{i} \geqslant 1$ units. The function $V(y)$ becomes more complicated, and the problem is now NPhard: For $p_{i}=1$ we have the standard knapsack problem. Furthermore, in this situation computing $V(y)$ is actually \#P-complete (Platzman et al. 1988). An interesting question is whether the techniques derived in this paper may be adapted to design approximation algorithms for this more general problem.

Another possible extension is to consider a multistage problem as in revenue management. In this situation the short-term assumption is no longer valid, and one may address offers in several rounds, maybe a fixed number $k$. One would now look for an adaptive online algorithm that, given the current situation and the number of rounds left, computes the optimal subset of clients to whom the offer should be addressed in the next round.

A third direction, related to the airline-industry application mentioned in $\S 1$, concerns pricing. When clients can be contacted in a personalized fashion by email, the airline may quote a different price for each selected client. Naturally, the clients' probability of accepting an offer will decrease as the price increases, while the value of a client increases with the selling price. Therefore, a natural model is to associate with client $i$ a decreasing function $p_{i}: \mathbb{R}_{+} \rightarrow$ $[0,1]$ and an increasing function $v_{i}: \mathbb{R}_{+} \rightarrow \mathbb{R}_{+}$, representing the acceptance probability and value for a given price, respectively. One may argue about the actual shape of these functions; for instance, it is natural to take $v_{i}(\cdot)$ as an affine function. The problem is now to select the customers to whom the offer will be made available, together with a price for each selected customer, so as to maximize the expected revenue.

\section{Appendix: Numerical Experiments}

In this section we present some statistics that illustrate the numerical performance of the algorithms $A_{1}^{*}$ through $A_{4}^{*}$ discussed previously. We also compare these solutions with two additional heuristics that work well in practice. The first heuristic starts from $A=\phi$ and recursively adds or removes a single element to get a maximal increase in value, namely
Heuristic 5 (IN-OUT)

Initialize $A_{5}^{*}=\phi$

Find $B$ with $\left|B \Delta A_{5}^{*}\right|=1$ and $V_{B}$ maximal

If $V_{B} \leqslant V_{A_{5}^{*}}$, then stop; otherwise set $A_{5}^{*}=B$ and repeat.

The resulting solution may be suboptimal, as for $m=1$ and $n=3$ with $p_{1}=0.5, p_{2}=0.9, p_{3}=1, v_{1}=75, v_{2}=50$, $v_{3}=47$, we have $A_{5}^{*}=\{1,3\}$ while the optimal solution is $A^{*}=\{1,2\}$. This example reveals another unexpected feature: If $A^{k} \subseteq C$ denotes an optimal solution for the original problem with the additional constraint $|A| \leqslant k$, then a customer included in $A^{k}$ need not be considered when we relax to $|A| \leqslant k+1$. As counterintuitive as it may seem, this occurs in the example above where $A^{1}=\{3\}$ while $A^{2}=A^{3}=A^{*}=\{1,2\}$.

The second heuristic builds on the previous idea considering not only the addition/removal of elements but also the swapping of an element in the current solution with an element from outside.

\section{Heuristic 6 (sWAP)}

Initialize $A_{6}^{*}=\phi$

Find $B$ of the form $B=\left(A_{6}^{*} \backslash\{i\}\right) \cup\{j\}$ with $V_{B}$ maximal If $V_{B} \leqslant V_{A_{6}^{*}}$, then stop; otherwise set $A_{6}^{*} \leftarrow B$ and repeat.

The resulting solution $A_{6}^{*}$ is still suboptimal as for $m=1$ and $n=5$ with

\begin{tabular}{lcccccc}
\hline Probabilities & $\left(p_{i}\right)$ & 0.7 & 0.6 & 0.7 & 0.2 & 1.0 \\
Values & $\left(v_{i}\right)$ & 94 & 69 & 68 & 67 & 64 \\
\hline
\end{tabular}

where $A_{6}^{*}=\{1,5\}$, while the optimal solution is $A^{*}=$ $\{1,2,3\}$.

Table A.1. Algorithm performance: $m=1, n=5$, 10,000 instances

\begin{tabular}{llrccr}
\hline Algorithm & & \% opt & Min ratio & Avg ratio & Time \\
\hline optimal & $\left(A^{*}\right)$ & 100.0 & 1.0000 & 1.0000 & 12.9 \\
MAX- $k$ & $\left(A_{1}^{*}\right)$ & 98.2 & 0.9590 & 0.9999 & 3.2 \\
ADD- $m$ & $\left(A_{2}^{*}\right)$ & 77.4 & 0.7531 & 0.9928 & 0.6 \\
MAX-AVG & $\left(A_{3}^{*}\right)$ & 51.2 & 0.7358 & 0.9793 & 0.7 \\
LP-RELAX & $\left(A_{4}^{*}\right)$ & 88.3 & 0.9451 & 0.9989 & 18.3 \\
IN-OUT & $\left(A_{5}^{*}\right)$ & 99.8 & 0.9489 & 0.9999 & 3.9 \\
SWAP & $\left(A_{6}^{*}\right)$ & 100.0 & 0.9972 & 1.0000 & 6.9 \\
\hline
\end{tabular}

Table A.2. Algorithm performance: $m=1, n=10,200$ instances.

\begin{tabular}{lcrccr}
\hline Algorithm & & \% opt & Min ratio & Avg ratio & Time \\
\hline optimal & $\left(A^{*}\right)$ & 100.0 & 1.0000 & 1.0000 & $1,611.2$ \\
MAX- $k$ & $\left(A_{1}^{*}\right)$ & 93.0 & 0.9916 & 0.9998 & 23.5 \\
ADD- $m$ & $\left(A_{2}^{*}\right)$ & 47.5 & 0.8168 & 0.9771 & 0.4 \\
MAX-AVG & $\left(A_{3}^{*}\right)$ & 11.5 & 0.8169 & 0.9564 & 2.3 \\
LP-RELAX & $\left(A_{4}^{*}\right)$ & 74.0 & 0.9775 & 0.9988 & 21.5 \\
IN-OUT & $\left(A_{5}^{*}\right)$ & 99.0 & 0.9918 & 0.9999 & 15.4 \\
SWAP & $\left(A_{6}^{*}\right)$ & 100.0 & 1.0000 & 1.0000 & 36.5 \\
& & & & &
\end{tabular}


Table A.3. Algorithm performance: $m=3, n=10,200$ instances.

\begin{tabular}{lcrccr}
\hline Algorithm & & \% opt & Min ratio & Avg ratio & Time \\
\hline optimal & $\left(A^{*}\right)$ & 100.0 & 1.0000 & 1.0000 & $1,601.8$ \\
MAX- $k$ & $\left(A_{1}^{*}\right)$ & 88.0 & 0.9813 & 0.9995 & 23.5 \\
ADD- $m$ & $\left(A_{2}^{*}\right)$ & 72.5 & 0.9049 & 0.9968 & 5.0 \\
IN-OUT & $\left(A_{5}^{*}\right)$ & 97.0 & 0.9738 & 0.9995 & 66.9 \\
SWAP & $\left(A_{6}^{*}\right)$ & 100.0 & 1.0000 & 1.0000 & 202.2 \\
\hline
\end{tabular}

We run a series of 10,000 random instances for $m=1$ and $n=5$ (with $p_{i}$ s and $v_{i}$ s generated uniformly in $[0,1]$ and $[0,100]$, respectively), which we solved exactly as well as by each of the six algorithms. Table A.1 shows the percentage of problems solved to optimality by each method, the minimal and average optimality ratios attained over the 10,000 trials, and the average running time per instance (in milliseconds).

The lower bound $\tilde{V}$ was always in the range $[0.5,0.86]$ with an average of 0.66 , while the upper bound $\bar{V}$ was in $[1.00,1.49]$ with 1.18 as average. The exercise was repeated with 200 instances of size $m=1$ and $n=10$, with the result shown in Table A.2.

We note the excellent performance attained by MAX- $k$ and LP-RELAX, at a small fraction of the execution time required by the brute force method. Despite the fact that ADD- $m$ and MAX-AVG are dominated by MAX- $k$, which finds the best threshold solution, these methods are still close to the optimum on average and are much faster, so they could be useful in applications where speed is an issue. On average, ADD- $m$ outperforms MAX-AVG both in optimal value and execution time, with the additional advantage that ADD- $m$ applies for all $m$ and not only for $m=1$ as MAX-AVG. The IN-OUT and SWAP heuristics get closer to the optimal value with a moderate increase in execution time. The situation is similar for $m=3, n=10$, as shown in Table A.3 (note that MAX-AVG and LP-RELAX do not apply for $m>1)$.

When we increase the dimension of the problem the exact solution takes too long to be computed by brute force and all the running times grow, with an increasing relative advantage for ADD- $m$. For $m=15$ and $n=50$ we get the following values and running times:

\begin{tabular}{llccc}
\hline Algorithm & MAX- $k$ & ADD- $m$ & IN-OUT & SWAP \\
\hline Value & $1,057.9$ & $1,057.9$ & $1,058.2$ & $1,058.2$ \\
Time & 3,265 & 140 & 41,740 & 494,321 \\
\hline
\end{tabular}

Note that the IN-OUT and SWAP running times increase significantly. We do not know if these heuristics run in polynomial time or not.

\section{Acknowledgments}

We thank two anonymous referees and the associate editor for many very helpful comments and suggestions. The first and second authors were partially supported by Instituto Milenio Sistemas Complejos de Ingeniería, and the last author was supported by FONDAP Matemáticas Aplicadas-CONICYT.

\section{References}

Ball, M., M. Queyranne. 2009. Toward robust revenue management: Competitive analysis of online booking. Oper. Res. 57 950-963.

Bult, J. R., T. Wansbeek. 1995. Optimal selection for direct mail. Marketing Sci. 14 378-394.

Chriqui, C., P. Robillard. 1975. Common bus lines. Transportation Sci. 9 $115-121$.

Cominetti, R., J. Vaisman. 2008. A sharp uniform bound for the distribution of a sum of Bernoulli random variables. Preprint, Departamento de Ingeniería Matemática, Universidad de Chile, Santiago, Chile.

Cormen, T., C. Leiserson, R. Rivest, C. Stein. 2001. Introduction to Algorithms. MIT Press, Cambridge, MA.

Dean, B., M. X. Goemans, J. Vondrak. 2008. Approximating the stochastic knapsack problem: The benefit of adaptivity. Math. Oper. Res. 33 945-964.

Jogdeo, K., S. M. Samuels. 1968. Monotone convergence of binomial probabilities and a generalization of Ramanujan's equation. Ann. Math. Statist. 39 1191-1195.

Littlewood, K. 1972. Forecasting and control of passengers. 12th AGIFORS Sympos. Proc. 95-128.

Platzman, L., J. Ammnos, J. Bartholdi III. 1988. A simple and efficient algorithm to compute tail probabilities from transforms. Oper. Res. 36 137-144.

Talluri, K. T., G. J. van Ryzin. 2005. The Theory and Practice of Revenue Management. Springer-Verlag, New York.

Siegel, A. 2001. Median bounds and their applications. J. Algorithms 38 184-236.

Vaisman, J. 2005. Convergencia fuerte del método de medias sucesivas para operadores lineales no-expansivos. Memoria de Ingeniería Civil Matemática, Universidad de Chile, Santiago, Chile. 